\title{
Improvements to the Overpotential of All-Solid-State Lithium-Ion Batteries during the Past Ten Years
}

\author{
Pilgun Oh, Hyomyung Lee, Seohyeon Park, Hyungyeon Cha, Junhyeok Kim, \\ and Jaephil Cho*
}

\begin{abstract}
After the research that shows that $\mathrm{Li}_{10} \mathrm{GeP}_{2} \mathrm{~S}_{12}$ (LGPS)-type sulfide solid electrolytes can reach the high ionic conductivity at the room temperature, sulfide solid electrolytes have been intensively developed with regard to ionic conductivity and mechanical properties. As a result, an increasing volume of research has been conducted to employ all-solid-state lithium batteries in electric automobiles within the next five years. To achieve this goal, it is important to review the research over the past decade, and understand the requirements for future research necessary to realize the practical applications of all-solid-state lithium batteries. To date, research on all-solid-state lithium batteries has focused on achieving overpotential properties similar to those of conventional liquid-lithium-ion batteries by increasing the ionic conductivity of the solid electrolytes. However, the increase in the ionic conductivity should be accompanied by improvements of the electronic conductivity within the electrode to enable practical applications. This essay provides a critical overview of the recent progress and future research directions of the all-solid-state lithium batteries for practical applications.
\end{abstract}

\section{Introduction}

Due to the rapid progress of energy storage systems using lithium-ion batteries (LIBs), ${ }^{[1-3]}$ the thermal instability of conventional liquid LIBs has led researchers to develop other types of lithium energy storage systems. For this reason, allsolid-state lithium batteries (ASSLBs) have been considered as the most promising candidates for high thermal stability, because their overall systems are composed of inorganic solid materials with low combustible properties. ${ }^{[4-6]}$ In this respect, the practical applicability of ASSLBs has been con-

\section{Prof. P. Oh, S. Park}

Department of Graphic Arts Information Engineering

Pukyong National University

Busan 485471, Republic of Korea

H. Lee, H. Cha, Dr. J. Kim, Prof. J. Cho

Department of Energy Engineering

School of Energy and Chemical Engineering

Ulsan National Institute of Science and Technology (UNIST)

Ulsan 44919, Republic of Korea

E-mail: jpcho@unist.ac.kr

The ORCID identification number(s) for the author(s) of this article can be found under https://doi.org/10.1002/aenm.202000904.

DOI: 10.1002/aenm.202000904 tinuously verified via the development of solid electrolytes (SEs) and appropriate active materials, which have high ionic conductivities and high applicability to ASSLBs systems, respectively. Unlike conventional liquid LIBs, which utilize organic liquid electrolytes as lithium ions and electron transfer passages, ASSLBs use inorganic solid SEs for the same purposes. Thus, the ionic conductivities of SEs play a critical role in increasing electrochemical performance during cell tests. In this respect, the research approaches for SEs have mainly focused on increasing their ionic conductivities. Because of that reason, a variety of inorganic SEs have been developed (e.g., oxides, polymers, and sulfides). ${ }^{[7-13]}$ Before 2010, the lithium ion conductivities of various SEs were much lower than the corresponding values of the liquid electrolytes. However, recent significant improvements in the ionic conductivities of SEs have resulted in novel designs of chemical elements, doping, and morphological control. ${ }^{[14-16]}$ Among them, oxide, polymer, and sulfide SEs have been intensively developed because of their high ionic conductivities and mechanical advantages. ${ }^{[17-22]}$ In particular, the recently developed sulfide SEs show high lithium ion conductivity, similar to the ionic conductivity of organic liquid electrolytes. Along with the development of SEs, much effort has been expended toward increasing the interfacial area and decreasing the side reactions between the SEs and the active materials via coatings and morphological control. ${ }^{[23-27]}$ However, although the recently developed SEs have achieved high ionic conductivities, the electrochemical performance of the ASSLBs and power density have not reached commercialization standards compared to the case of conventional liquid LIBs. Given the low electrochemical performance of the ASSLBs in spite of the high ionic conductivities, the overall overpotential and electronic conductivity within the battery should be emphasized because it could affect the electrochemical performance during cell tests. Herein, we review recent progress in ASSLBs and their battery performance in terms of the materials and electrodes. In addition, we propose a future research direction for increasing the electrochemical performance and improving the overall overpotential property via novel electrode design. 


\section{Previous Research in All-Solid-State Lithium Batteries}

In general, the electrodes of the ASSLBs are composed of active materials, current collectors, conducting agents, and SEs. Among all the compositions of the ASSLBs, the ionic conductivity of the SE is considered to be the most important factor which could affect the electrochemical performance of the ASSLBs because it is directly related to the lithium ion transfer. For this reason, to date, many researchers have concentrated on identifying and developing SEs with high ionic conductivities. As shown in Figure 1a, the ionic conductivity of SEs has increased extensively over the past ten years. ${ }^{[7-13,18,28-34]}$ In particular, the development of $\mathrm{Li}_{10} \mathrm{GeP}_{2} \mathrm{~S}_{12}$ (LGPS) sulfide $\mathrm{SEs}$ in 2011 by Kanno and co-workers opened new prospects from the commercialization point of view. ${ }^{[11]}$ Before the development of LGPS-type sulfide electrolytes, the ionic conductivities of the SEs were barely comparable to those of liquid organic electrolytes at high temperatures $\left(50-80^{\circ} \mathrm{C}\right)$. However, LGPS-phase SEs have extremely high ionic conductivities at room temperature $(12 \mathrm{mS}$ $\mathrm{cm}^{-1}$ ), which is helpful for increasing the electrochemical performance of the ASSLBs. After that, researchers have developed improved LGPS-type sulfide SEs via doping or composition change. ${ }^{[7,10,35]}$ In addition, the development of argyrodite-type sulfide SEs, which have high ionic conductivities, contributed to expanding the practical applicability of ASSLBs. ${ }^{[12,36]}$ Besides the development of various types of SEs, many studies have concentrated on manufacturing techniques of SEs for largescale processes. For example, the liquid-phase synthesis process of SEs is considered to be an appropriate method for reducing the manufacturing time and scale up. ${ }^{[14,32,37]}$ The recent SEs synthesized by the liquid phase process have ionic conductivities similar to that provided by the original mechanical synthesis process. Although research on SEs has resulted in successful outcomes with respect to the ionic conductivity, synthesis method, and morphological control, the electrochemical performance of ASSLBs which uses transition metal oxide as active materials still suffers from large gaps compared to that of conventional liquid LIBs. As shown in Figure 1a, the specific capacities of the ASSLBs have not increased extensively during the last ten years of research in this area. ${ }^{[26,38-52]}$ More notably, a large specific capacity gap exists between the ASSLBs and conventional LIBs even though the ionic conductivity of SEs is similar to those of liquid electrolytes. Furthermore, as shown in Figure 1b, while electrochemical tests on conventional LIBs have been conducted at various C-rates, such as $1 \mathrm{C}, 2 \mathrm{C}, 5 \mathrm{C}$, and 10C, most ASSLBs have been tested with a low C-rate of 0.1C. ${ }^{[24,47,53-58]}$ More practically, the ASSLBs show extensively low specific capacities when it tested at a high C-rate such as 1C. However, conventional LIBs show high specific capacities even when they are tested at the high C-rates of $5 \mathrm{C}$ and $10 \mathrm{C}$ In addition, the electrode of the ASSLBs which is the critical factor of deciding the energy density still has challenges such as active material ratio, loading level, and fabrication method. In case of the ASSLBs, most previous research has employed the dry mixing electrode fabrication method which is inappropriate for scale up because of its mechanical instability. However, the conventional liquid LIBs have employed the wet slurry mixing electrode fabrication method which is appropriate for scale up

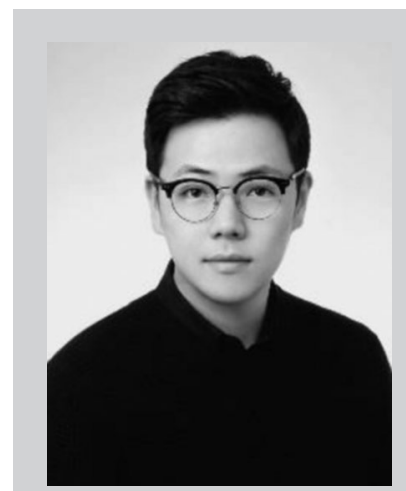

Pilgun Oh is a professor in department of Graphic Arts Information Engineering at Pukyong National University (Republic of Korea). He recieved his Ph.D degree in Energy Engineering (Battery science and technology) from Ulsan National Institute of Science and Technology (UNIST) in 2015. He performed the postdoctoral study at the University of Texas at Austin (USA) under supervision of Prof. Arumugam Manthiram. He studied all-solid state lithium ion batteries at Hyundai Motors. His current research is focused on material engineering of cathode materials and system/ electorde design for Li-ion and all-solid state Li-ion batteries.

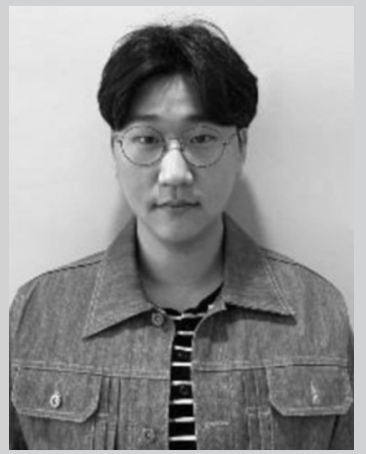

HyoMyung Lee received his B.S. degree in Energy and Chemical Engineering from Ulsan National Institute of Science and Technology (UNIST) in Republic of Korea. He is currently pursuing his Ph.D. degree in School of Energy and Chemical Engineering at UNIST under the supervision of Prof. Jaephil Cho since 2017. His research interests include the development and investigation of the layered cathode materials for Li-ion and all-solid state Li-ion batteries.

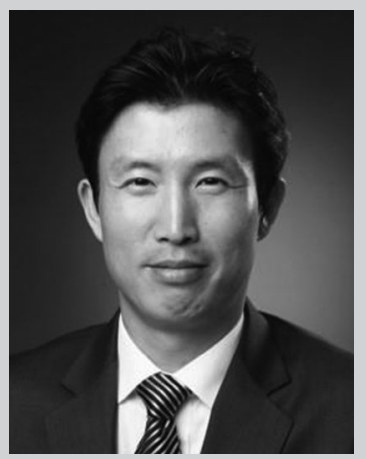

Jaephil Cho is a distinguished professor of school of energy and chemical engineering at UNIST (Republic of Korea). $\mathrm{He}$ is a director of Samsung SDI-UNIST Future Battery Research Center. His current research is focused mainly on Li-ion, all-solid state Li-ion, metal-air, and redox flow batteries for energy storage.

and make good contact area between the active material and the other composition materials within the electrode. More importantly, to attain high energy density, most researchers of the conventional liquid LIBs not only increased the active material ratio of the electrode, but also raised its loading level of the electrode. 


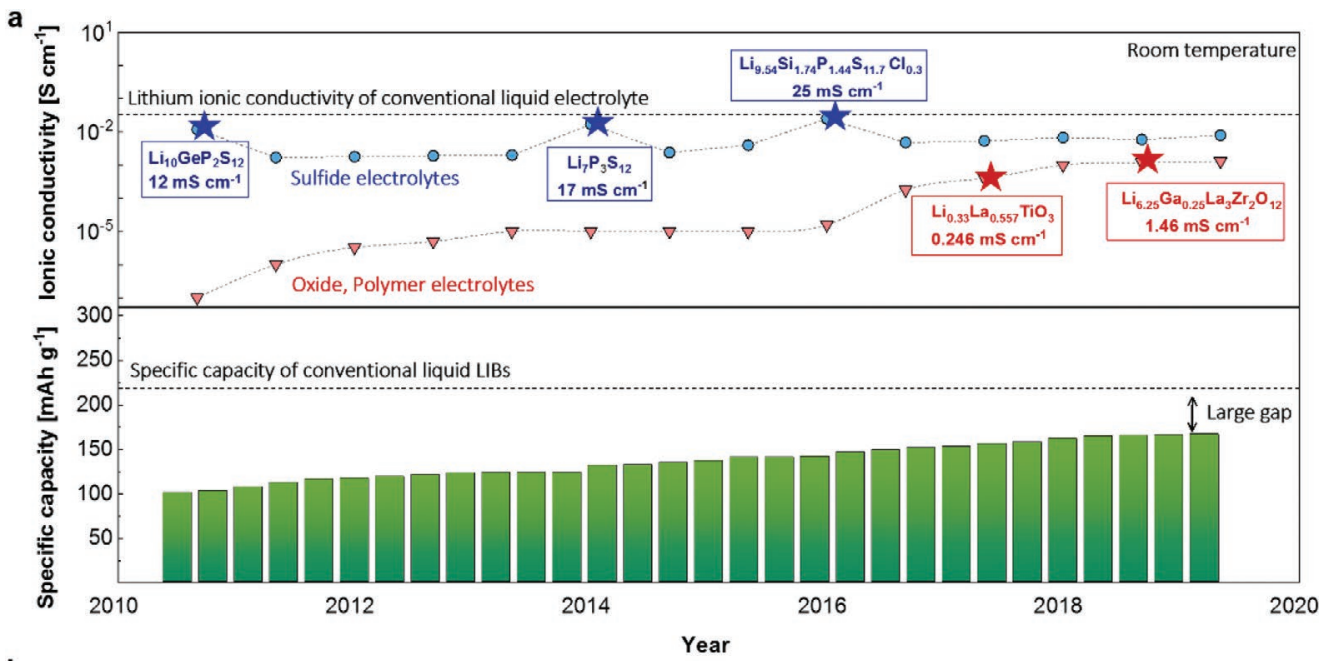

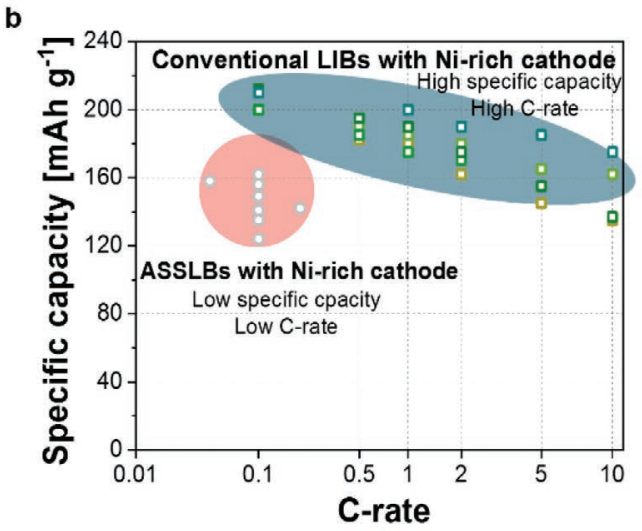

d

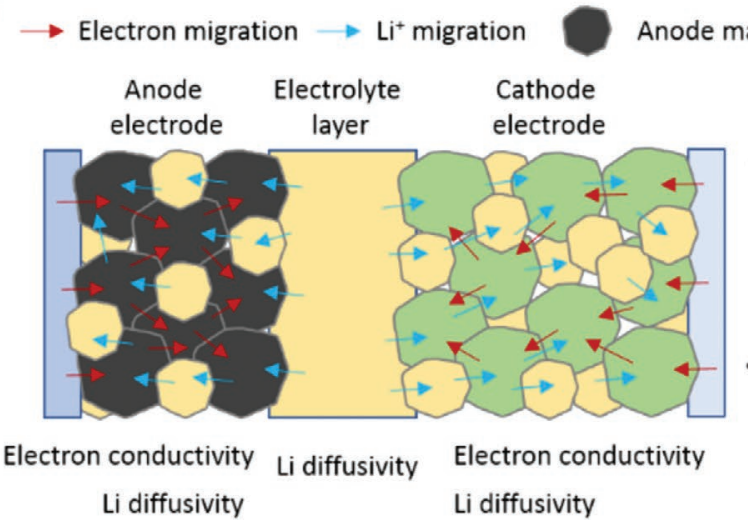

Li diffusivity Li diffusivity

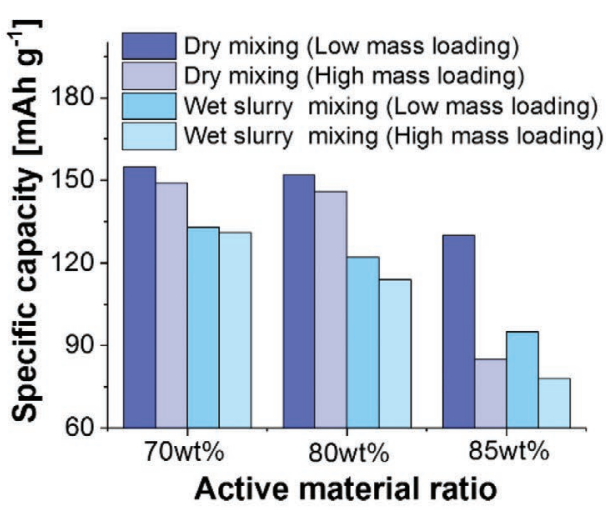

Electrolyte

Cathode material

- Interfacial electron $/ \mathrm{Li}^{+}$resistance btw active material and electrolyte

- Bulk electron/Li+ resistance within active material

- Bulk electron/Li+ resistance within electrolyte

Figure 1. Previous research of the ASSLBs. a) Development of the oxide, polymer, and sulfide solid electrolytes and previous reported specific capacity of the all-solid-state lithium batteries (ASSLBs) during past ten years. b) Previous reported specific capacity of the ASSLBs and the conventional lithium ion batteries (LIBs) with regard to its various current density. c) Specific capacity comparison with different active material ratio, electrode fabrication method and mass loading level. c) Reproduced with permission. ${ }^{[26]}$ Copyright 2018, Elsevier. d) Schematic of the ASSLBs which is composed of cathode, anode, and the solid electrolytes (SEs) with its internal and interfacial resistance.

However, as shown in Figure 1c, the ASSLBs exhibit lower specific capacity when the wet slurry electrode fabrication method is used, compared to those produced by the dry mixing electrode fabrication method. ${ }^{[26]}$ Furthermore, when the active material ratio is increased from 70 to $85 \mathrm{wt} \%$, the specific capacity of the ASSLBs shows almost $61 \%$ capacity fading with the wet slurry electrode fabrication method at high mass loading levels. These challenges with regard to the low specific capacity, low current density, and mild electrode condition have hindered the practical application of ASSLBs. To resolve these critical problems, it is important to focus on the ionic and electronic conductivities within the electrode of the ASSLBs, which are critical factors of capacity and power density. For example, Figure 1d shows an overall ASSLBs scheme composed of the SE layer and the active material electrode layer. During electrochemical testing, the lithium ions and electrons should pass through the interface 
between the active materials and the SEs, the active material, and the SEs itself. Therefore, interfacial resistance between the active materials and the SEs is inevitable. In addition, the internal resistances of the bulk active materials and the SEs are also important factors. Therefore, although the lithium bulk ionic conductivities of the SEs are almost similar to the ionic conductivity of the liquid electrolyte, they could be decreased by the interfacial electron/lithium resistance between the active material and the SEs, the bulk electron/lithium resistance within the active material, and the SEs themselves. Given these internal and interfacial resistances of the ASSLBs, the specific capacity and power density could not match the high lithium ionic conductivities of the SEs. As a result, for practical applications of ASSLBs, it is important to focus on decreasing the interfacial and bulk resistances of the ASSLBs and increasing the electronic conductivity.

\section{Recent Progress: Surface Control and Novel Electrode Design Process to Improve Lithium Ion Conductivity of ASSLBs Electrode}

We have addressed the recent progress and prospects in the ASSLBs (Figure 2a). As mentioned above, to increase the electrochemical performance, it is necessary to consider the occurrence of overall overpotential within the batteries because of the interfacial and internal resistances of the ASSLBs. In this regard, researchers have focused on introducing a buffer layer on the surface of the active materials, which is helpful in the formation of a facile lithium pathway. In addition, such a buffer layer could play a large role in preventing the side reaction between the SE layer and the active materials. During the past decade, various types of materials, such as transition metal oxides, carbon materials, and SEs, have been applied as buffer

a

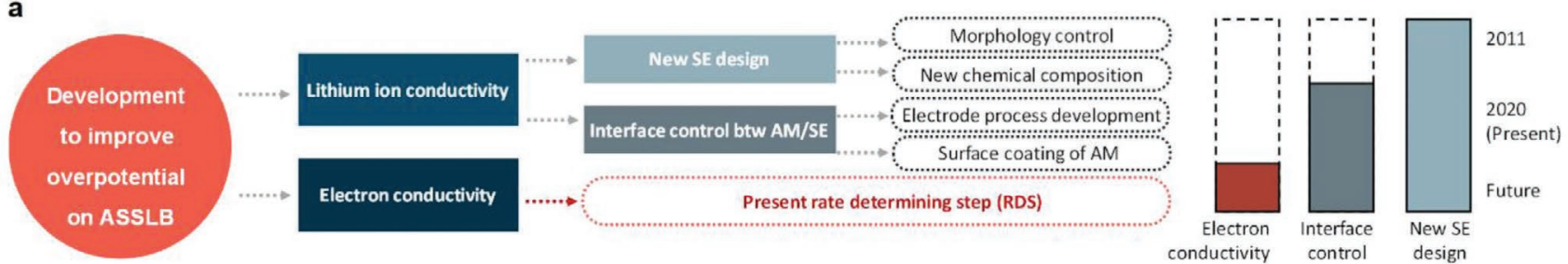

b

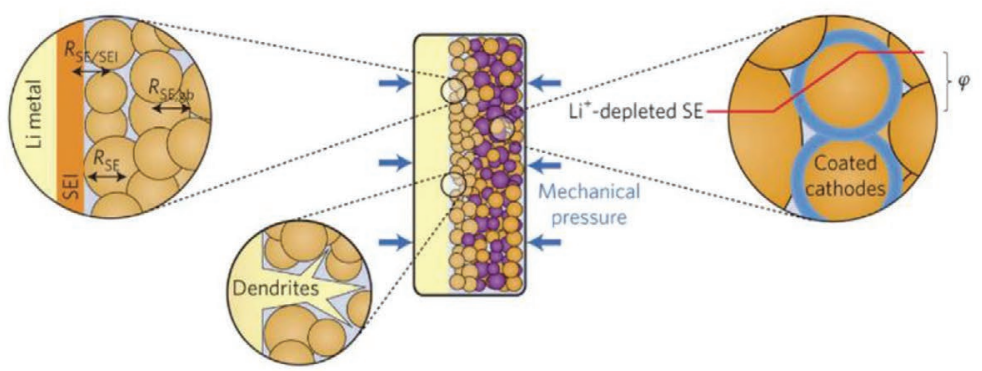

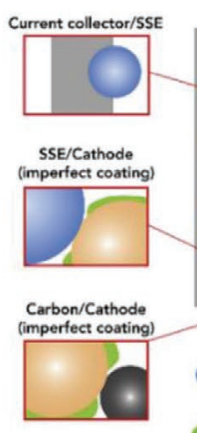

conductivit

control design
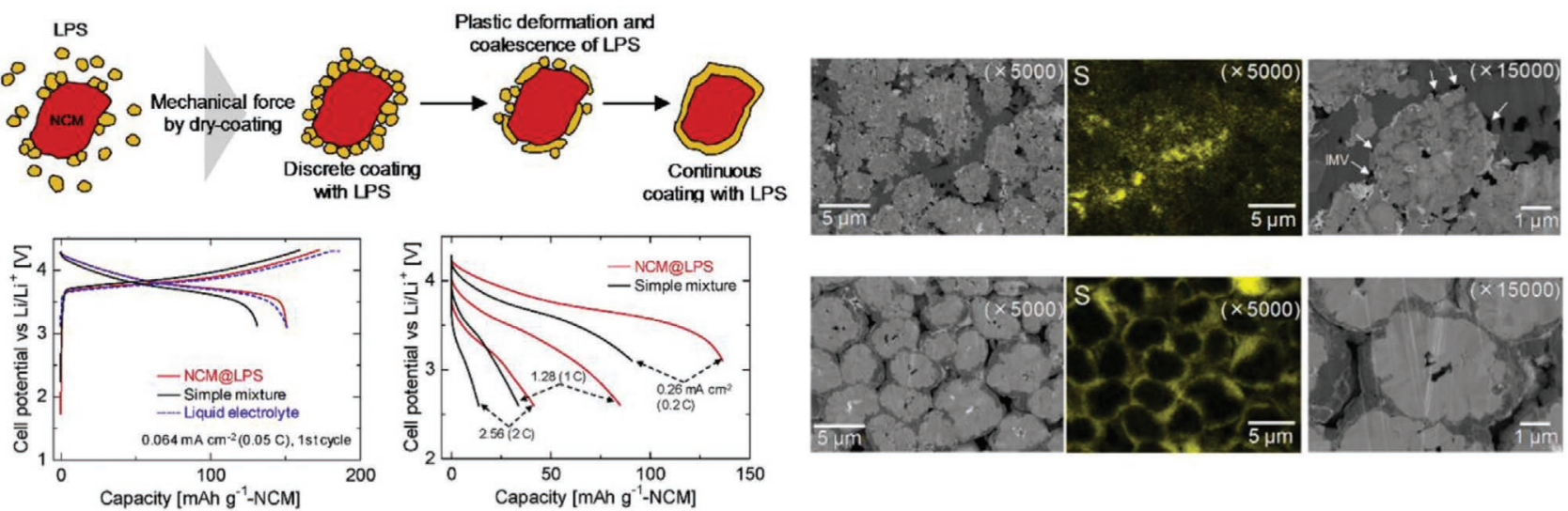

Figure 2. Recent research for alleviate the interfacial resistance of the ASSLBs. a) Schematic illustration of recent progress and challenges of the ASSLBs. b) Schematic of possible interfacial resistance area within the conventional ASSLBs. b) Reproduced with permission. [65] Copyright 2016, Springer Nature. c) Possible interfacial area of the ASSLBs in the cathode electrode. c) Reproduced with permission. ${ }^{[66]}$ Copyright 2019, Elsevier. d) Schematic of process of the $\mathrm{SE}$ coating by dry-coating, first charge-discharge voltage profiles of Ni based layered oxides (NCM) bare (simple mixture), $\mathrm{Li}_{3} \mathrm{PS}{ }_{4}$ Coated NCM (NCM@LPS) and liquid electrolyte half cells, discharge curves of NCM@LPS and NCM bare (simple mixture) at various current densities. e) Cross-sectional scanning electron microscopy (SEM) image and EDS analysis of the SE coated NCM and bare NCM d,e) Reproduced with permission. ${ }^{[68]}$ Copyright 2020, Elsevier. 
layers, and have been found to be appropriate for ASSLBs systems. ${ }^{[39,59-64]}$ However, although these buffer layers could effectively decrease the interfacial resistance and form a facile lithium pathway, the current electrochemical performance of ASSLBs is still lower than the electrochemical performance of the conventional liquid LIBs. As a result, previously reported research related to buffer layers could not solve the intrinsic problems of the interfacial and internal resistances within the electrode. In this respect, it is essential to identify the overall formation and propagation mechanisms of the interfacial and internal resistances within the ASSLBs for successful application of the buffer layer. As shown in Figure 2b, with respect to the overall cell architecture, many factors can increase the interfacial resistance within the electrode. ${ }^{[65]}$ In the case of the lithium metal anode, the formation of an solid electrolyte interface (SEI) layer between the lithium anode and the SE increases the overpotential, which is harmful to cell performance. In addition, the resistive interface between each SE should be considered, because it could also increase the overpotential of the ASSLBs. In case of the cathode materials, the buffer layer has been considered as crucial for decreasing the side reactions with the SEs. Moreover, the buffer layer is helpful to avoid the potential lithium depletion area at the cathode materials. In addition, Xiao and co-workers reported various interface situations in a coated cathode composite and an ideal coating strategy using a computational framework, as shown in Figure 2c. ${ }^{[66]}$ They reported certain advantages and disadvantages between imperfect and perfect coatings on the surface of the cathodes. In case of the cathodes with imperfect coatings, capacity fading was evident because of the direct contact between the uncoated surface and the SEs. However, cathodes with imperfect coatings exhibited direct contact between the cathode and the conducting additives, resulting in an increase of electronic conductivity. On the other hand, perfectly coated cathodes could prevent direct contact between the SEs and decrease the side reaction, but the electronic conductivity would also decrease because of the low contact with the conducting additives. As a result, it is necessary to develop novel cathode materials that could satisfy the requirements of both chemical stability and high electronic conductivity. In addition, as shown in Figure 2c, the interface of the conducting additives should be carefully controlled because oxidation of the SEs could occur at that interface, resulting in increase of the interfacial resistance. ${ }^{[66,67]}$ In particular, at a low current density, the interface between the conducting additives and the SEs is not directly involved with lithium ion transfer and electronic conductivity. However, it could be transformed into an electrochemically inactive area at a high current density, resulting in a resistive barrier. Considering the previously reported data in Figure 2c, the interfaces between the active materials and the SEs, conducting additives, and the SEs should be modified and developed for facile lithium and electron transfer during electrochemical testing. Notably, Xiao and co-workers also stated that the coating material needs to be carefully selected according to the specific SE type and cathode interface. ${ }^{[6]}$ Therefore, with regard to the electrode making process, cell making process, and various type of the SEs, the appropriate coating materials could be different depending on the differences in the reactivity and oxidation limits between the SEs and active materials.
Although an appropriate approach for coating material selection with regard to the SEs and active materials could help increase the chemical stability and ionic conductivity of the electrode, another process should be devised to increase the electronic and ionic conductivities of the electrode via a novel electrode design. For example, other approaches have been made to decrease the dead space between the active materials and the SEs as previously mentioned before by introducing the SE coatings on the surface of the active materials. ${ }^{[60,68]}$ As shown in Figure 2d, Nakamura et al. produced a uniform coating layer of $\mathrm{Li}_{3} \mathrm{PS}_{4}$ (LPS) on the surface of $\mathrm{LiNI}_{1 / 3} \mathrm{Co}_{1 / 3} \mathrm{Mn}_{1 / 3} \mathrm{O}_{2}$ (NCM) by using the dry coating methods. ${ }^{[68]}$ During the coating process, unlike the other coating method which are complex and low efficiency, they made the core-shell particles which has SE shell and the active materials core by simple dry mixing of powder with mechanical forces. This core-shell type active materials have advantages in retaining its secondary type morphology of nickel-rich cathode even when there is high pressure during the cell fabrication process. In addition, it is helpful to decrease the dead space due to the softness and ductility of the sulfide SEs. To identify the decrease of the dead space, they conducted the cross-sectional scanning electron microscopy (SEM) analysis of electrode between the uncoated and LPS coated particles. As shown in Figure 2e, in case of the electrode with uncoated cathode materials, there are many cracks and micro-voids which are formed during electrode and cell fabrication process. However, in the case of the electrodes which are composed of the core-shell composite, they retained their initial secondary particle shape and made low ratio of micro-voids. In addition, they analyzed the 3D internal structure of the core-shell cathode composite by the focused ion beam-scanning electron microscope (FIB-SEM) system. Compared to the electrode with uncoated cathode composite, the inter- and intra-void fraction and contact area of core-shell cathode composite are low, resulting in facile lithium path and electron path. As a result, during the first charge and discharge electrochemical test, LPS-coated cathode materials show high discharge capacity of $160 \mathrm{mAh} \mathrm{g}^{-1}$ which is similar to that of the liquid electrolyte half-cell. In addition, it shows outstanding electrochemical performance even when the cell was tested at a C-rate of $0.2 \mathrm{C}, 1 \mathrm{C}$, and $2 \mathrm{C}$. These approaches show solid electrolyte coating on cathode surface not only increased the ionic and electronic conductivity of the electrode but also decreased the ratio of electrochemical inactive area (Figure 2d).

In addition to the SE coating on the cathode surface, there have been many efforts for increasing contact area and ionic conductivity via changing the electrode fabrication process. Among the variety of efforts, Kim et al. designed the new type of electrode via infiltration SE solutions. ${ }^{[9]}$ Moreover, they infiltrated the SEs solution into the electrode of conventional liquid LIBs which has high active material ratio. The ASSLBs used the SE-infiltrated electrode which shows outstanding electrochemical performance which is similar to the electrochemical performance of the conventional liquid LIBs. Considering the electrode used in electrochemical test has high active material ratio in the electrode related to the high energy density, this approach should be emphasized because it opens a new prospect of the ASSLBS with respect to the electrode design. 


\section{Future Perspectives: Importance of Electric Conductivity on ASSLBs}

As mentioned earlier, research on ASSLBs has focused on the development of SEs to replace conventional liquid electrolytes, and most of these studies have targeted improving the low electrode lithium ion conductivity. However, despite these noticeable advances, compared to liquid conventional LIBs, the ASSLBs still exhibit poor electrochemical performance in terms of electrode energy density, rate property, and cycle performance.

Considering the poor performance (e.g., in rate capability) despite improved lithium ion conductivity, it is evident that electrical conductivity within the electrode plays a crucial role in this issue (Figure 2a). Figure 3a,b shows the electrode crosssections for an ASSLBs and a conventional liquid LIBs. When comparing the distribution of the conductive material (CM) in each electrode, the CMs within the ASSLBs electrode are severely agglomerated in places, whereas the $\mathrm{CM}$ of the conventional LIBs electrode shows excellent dispersity. Note that in the case of ASSLBs, nonpolar solvents such as gylene and heptyl butyrate are applied during electrode preparation because sulfide-based electrolytes cause side reactions with polar solvents due to their chemical instability. Unfortunately, it is widely known that conductive materials based on carbon materials have very poor dispersibility in nonpolar solvents. Moreover, owing to the high interface resistance between the SE and the active material, most ASSLBs electrodes are fabricated with a high proportion of electrolytes, which are electronic insulators. In other words, compared to conventional liquid LIBs, the electronic conductivity of ASSLBs could be insufficient due to the poor dispersibility of the CMs and a high electrolyte ratio. a

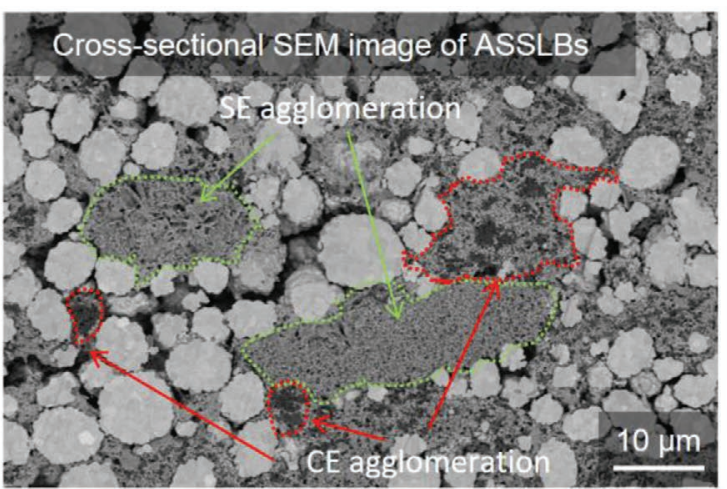

b

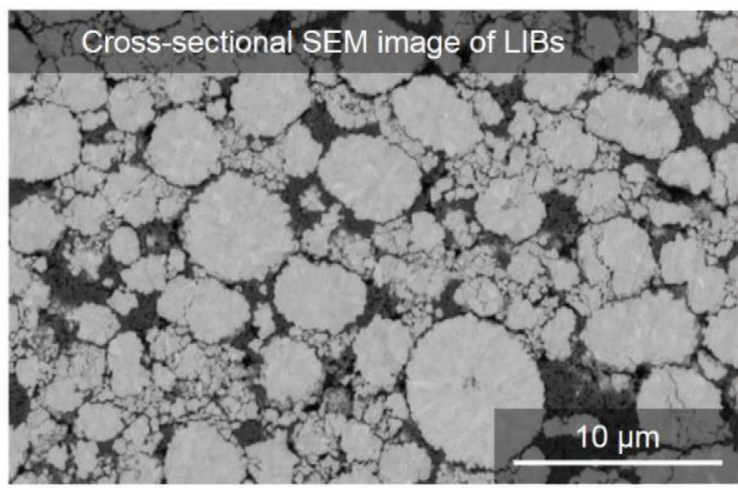

c $\rightarrow$ Electron migration $\rightarrow \mathrm{Li}^{+}$migration $\bigcirc$ Electrolyte $\bigcirc$ Cathode material $\bigcirc$ Conductive material

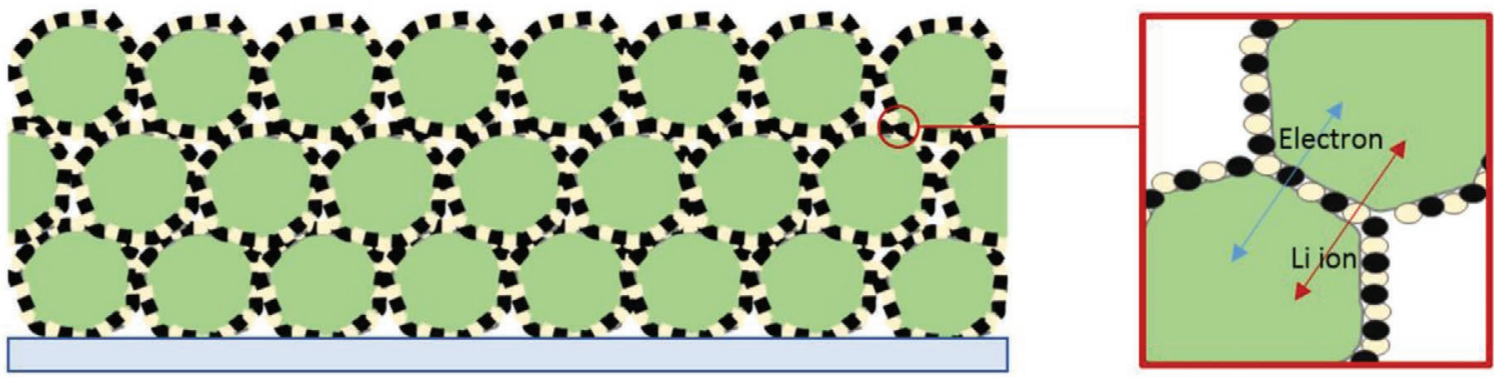

d

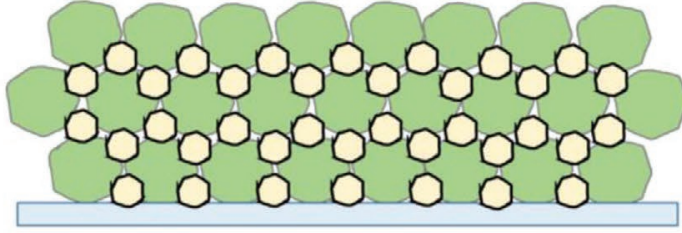

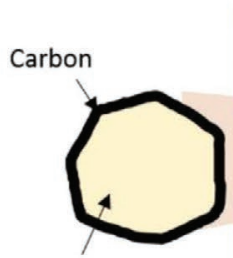

Electrolyte

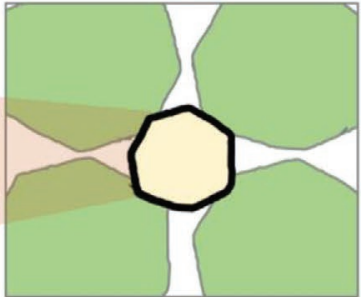

Before press process

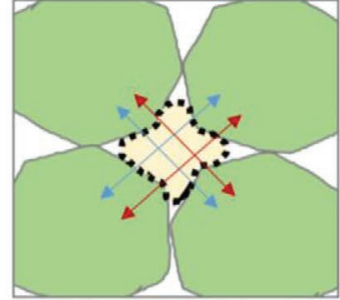

After press process

Figure 3. Cross-sectional SEM image of a) the ASSLBs and b) the conventional liquid LIBs. c) Schematic illustration of the rational electrode of ASSLBs d) Schematic illustration of the possible future research direction to decrease the over potential of the overall ASSLBs. 
Therefore, even if the lithium ion conductivity of the electrode is improved, ASSLBs face performance limitations arising from the lack of electron pathways.

Accordingly, it is very important to investigate improvements to increase the electronic conductivity. Thus, how can we effectively improve the electronic conductivity of ASSLBs? We believe that the electrode shown in Figure $3 \mathrm{c}$ presents a rational electrode design. This electrode design suggests that active materials' surface should be covered by CMs and partially alternated with SEs, rather than using an encapsulated active material with the SE. Such an arrangement will improve the conductivities of both the lithium ion and the electron. Figure $3 \mathrm{~d}$ demonstrates an experimentally obtainable ideal electrode design. To realize the experimental concept, a nano-thickness carboncoated SE is essential. As the general purpose of the electrolyte in the battery system is to transport lithium ions and prevent electron migration, this material engineering may seem contradictory. However, note that the electrolyte within the cathode or anode electrode should exhibit multiple functions of superior electronic conductivity as well as high lithium ion conductivity. The newly designed ASSLBs electrode is composed of ew carbon coated SE and active materials, realizing a bimodal electrode configuration (Figure $3 \mathrm{~d}$ ). As the following press process is conducted during cell fabrication process, the morphology of the carbon-coated electrolyte changes, and the surface carbon coating layer cracks, resulting in an electrolyte surface that is covered with carbon material partially alternated with the SE. This electrode engineering effectively fills the electrode voids with electrolytes and disperses the lithium ion pathway as well as the electron pathway. The proposed experimental demonstration (Figure 3d) can effectively realize the superior electrochemical performance of the ideal electrode design in Figure 3c. In addition to the above electrode design, advanced material engineering, such as the development of nano-sized sulfide SEs, increased ductilities of the electrolyte and light electrolyte, and new electrode designs are also required to maximize the electronic conductivity of ASSLBs.

\section{Conclusion}

In this essay, we summarized the progress reported by recent studies and their limitations toward addressing the overpotential issue of all-solid-state batteries. In particular, many researchers have developed a variety of SE materials with superior ionic conductivity that is similar to that of liquid electrolytes. Unfortunately, despite the enhanced ionic conductivity of SEs, ASSLBs have shown electrochemical limits in terms of low energy density and poor rate performances. In this regard, recent studies have focused on improving the interfacial conductivity by increasing the contact between SEs and active materials. However, we suggest that an all-solid-state battery based on improving only ionic conductivity results in performance limits on the rate capability; thus, further approaches are required to enhance electronic conductivity in solid-state battery systems. To this end, a reasonable electrode concept, including a novel material design and electrode process, was introduced. This essay thus presents a progressive research approach on superior power properties for next-generation all-solid-state batteries. Furthermore, it provides innovative research directions and insights relevant to ASSLBs.

\section{Acknowledgements}

P.O. and H.L. contributed equally to this work. This work was supported by the National Research Foundation of Korea (NRF) grant funded by the Korea government (MSIT) (NRF-2020R1C1C1009641). Also, financial support from the 2020 Research Funds (1.200029.1) of Ulsan National Institute of Science and Technology (UNIST) is greatly acknowledged.

\section{Conflict of Interest}

The authors declare no conflict of interest.

\section{Keywords}

all-solid-state lithium batteries, electrode design, electronic conductivity, ionic conductivity, material engineering, overpotentials

Received: March 9, 2020

Revised: April 8, 2020 Published online:

[1] J. Kim, J. Lee, H. Ma, H. Y. Jeong, H. Cha, H. Lee, Y. Yoo, M. Park, J. Cho, Adv. Mater. 2018, 30, 1704309.

[2] N. Nitta, F. Wu, J. T. Lee, G. Yushin, Mater. Today 2015, 18, 252.

[3] A. Manthiram, B. Song, W. Li, Energy Storage Mater. 2017, 6, 125.

[4] H. Lee, P. Oh, J. Kim, H. Cha, S. Chae, S. Lee, J. Cho, Adv. Mater. 2019, 31, 1900376

[5] Z. Gao, H. Sun, L. Fu, F. Ye, Y. Zhang, W. Luo, Y. Huang, Adv. Mater. 2018, 30, 1705702

[6] S. Xia, X. Wu, Z. Zhang, Y. Cui, W. Liu, Chem 2019, 5, 753.

[7] F. Wu, W. Fitzhugh, L. Ye, J. Ning, X. Li, Nat. Commun. 2018, 9, 4037.

[8] K. Homma, M. Yonemura, T. Kobayashi, M. Nagao, M. Hirayama, R. Kanno, Solid State lonics 2011, 182, 53.

[9] J. F. Wu, W. K. Pang, V. K. Peterson, L. Wei, X. Guo, ACS Appl. Mater. Interfaces 2017, 9, 12461.

[10] Y. Kato, S. Hori, T. Saito, K. Suzuki, M. Hirayama, A. Mitsui, M. Yonemura, H. Iba, R. Kanno, Nat. Energy 2016, 1, 16030.

[11] N. Kamaya, K. Homma, Y. Yamakawa, M. Hirayama, R. Kanno, M. Yonemura, T. Kamiyama, Y. Kato, S. Hama, K. Kawamoto, A. Mitsui, Nat. Mater. 2011, 10, 682.

[12] Z. Zhang, L. Zhang, Y. Liu, C. Yu, X. Yan, B. Xu, L. M. Wang, J. Alloys Compd. 2018, 747, 227.

[13] M. Tachez, J.-P. Malugani, R. Mercier, G. Robert, Solid State lonics 1984, 14, 181.

[14] S. Teragawa, K. Aso, K. Tadanaga, A. Hayashi, M. Tatsumisago, J. Mater. Chem. A 2014, 2, 5095.

[15] N. H. H. Phuc, M. Totani, K. Morikawa, H. Muto, A. Matsuda, Solid State lonics 2016, 288, 240.

[16] C. Y. Eun, P. K. Ho, K. D. Hyeon, O. D. Yang, K. H. Ram, L. Young-Gi, J. Y. Seok, ChemSusChem 2017, 10, 2605.

[17] W. Zhou, Z. Wang, Y. Pu, Y. Li, S. Xin, X. Li, J. Chen, J. B. Goodenough, Adv. Mater. 2019, 31, 1805574.

[18] Z. Wan, D. Lei, W. Yang, C. Liu, K. Shi, X. Hao, L. Shen, W. Lv, B. Li, Q.-H. Yang, F. Kang, Y.-B. He, Adv. Funct. Mater. 2019, 29, 1805301. 
[19] Q. Zhang, D. Cao, Y. Ma, A. Natan, P. Aurora, H. Zhu, Adv. Mater. 2019, 31, 1901131.

[20] K. Kimura, K. Wagatsuma, T. Tojo, R. Inada, Y. Sakurai, Ceram. Int. 2016, 42, 5546.

[21] T. Thompson, S. Yu, L. Williams, R. D. Schmidt, R. Garcia-Mendez, J. Wolfenstine, J. L. Allen, E. Kioupakis, D. J. Siegel, J. Sakamoto, ACS Energy Lett. 2017, 2, 462.

[22] X. Ban, W. Zhang, N. Chen, C. Sun, J. Phys. Chem. C 2018, 122, 9852.

[23] T. Liu, Y. Zhang, X. Zhang, L. Wang, S. X. Zhao, Y. H. Lin, Y. Shen, J. Luo, L. Li, C. W. Nan, J. Mater. Chem. A 2018, 6, 4649.

[24] Y. Cao, Q. Li, S. Lou, Y. Ma, C. Du, Y. Gao, G. Yin, J. Alloys Compd. 2018, 752, 8

[25] F. Strauss, T. Bartsch, L. de Biasi, A. Y. Kim, J. Janek, P. Hartmann, T. Brezesinski, ACS Energy Lett. 2018, 3, 992.

[26] Y. J. Nam, D. Y. Oh, S. H. Jung, Y. S. Jung, J. Power Sources 2018 375, 93.

[27] F. Han, T. Gao, Y. Zhu, K. J. Gaskell, C. Wang, Adv. Mater. 2015, 27 3473.

[28] P. Zhu, C. Yan, M. Dirican, J. Zhu, J. Zang, R. K. Selvan, C.-C. Chung, H. Jia, Y. Li, Y. Kiyak, N. Wu, X. Zhang, J. Mater. Chem. A 2018, 6, 4279.

[29] M. A. Kraft, S. Ohno, T. Zinkevich, R. Koerver, S. P. Culver, T. Fuchs, A. Senyshyn, S. Indris, B. J. Morgan, W. G. Zeier, J. Am. Chem. Soc. 2018, 140, 16330.

[30] M. R. Busche, D. A. Weber, Y. Schneider, C. Dietrich, S. Wenzel, T. Leichtweiss, D. Schröder, W. Zhang, H. Weigand, D. Walter, S. J. Sedlmaier, D. Houtarde, L. F. Nazar, J. Janek, Chem. Mater. 2016, 28, 6152

[31] J.-F. Wu, E.-Y. Chen, Y. Yu, L. Liu, Y. Wu, W. K. Pang, V. K. Peterson, X. Guo, ACS Appl. Mater. Interfaces 2017, 9, 1542.

[32] Y. Wang, D. Lu, M. Bowden, P. Z. El Khoury, K. S. Han, Z. D. Deng, J. Xiao, J.-G. Zhang, J. Liu, Chem. Mater. 2018, 30, 990.

[33] K. Takada, J. Power Sources 2018, 394, 74.

[34] S. Li, S.-Q. Zhang, L. Shen, Q. Liu, J.-B. Ma, W. Lv, Y.-B. He, Q.-H. Yang, Adv. Sci. 2020, 7, 1903088.

[35] D. Xie, S. Chen, Z. Zhang, J. Ren, L. Yao, L. Wu, X. Yao, X. Xu, J. Power Sources 2018, 389, 140.

[36] S. Wang, Y. Zhang, X. Zhang, T. Liu, Y.-H. Lin, Y. Shen, L. Li, C.-W. Nan, ACS Appl. Mater. Interfaces 2018, 10, 42279.

[37] S. Chida, A. Miura, N. C. Rosero-Navarro, M. Higuchi, N. H. H. Phuc, H. Muto, A. Matsuda, K. Tadanaga, Ceram. Int. 2018, 44, 742.

[38] H. W. Kwak, Y. J. Park, Thin Solid Films 2018

[39] S. Ito, S. Fujiki, T. Yamada, Y. Aihara, Y. Park, T. Y. Kim, S.-W. Baek, J.-M. Lee, S. Doo, N. Machida, J. Power Sources 2014, 248, 943.

[40] A. Sakuda, T. Takeuchi, H. Kobayashi, Solid State Ionics 2016 285,112

[41] T. Onodera, J. Kawaji, A. Sato, T. Okumura, J. Ceram. Soc. Jpn. 2017 125, 494.

[42] H. Wakayama, Y. Kawai, J. Mater. Chem. A 2017, 5, 18816

[43] M. Yamamoto, M. Takahashi, Y. Terauchi, Y. Kobayashi, S. Ikeda, A. Sakuda, J. Ceram. Soc. Jpn. 2017, 125, 391.
[44] W. Zhang, D. A. Weber, H. Weigand, T. Arlt, I. Manke, D. Schröder, R. Koerver, T. Leichtweiss, P. Hartmann, W. G. Zeier, J. Janek, ACS Appl. Mater. Interfaces 2017, 9, 17835.

[45] R. Koerver, I. Aygün, T. Leichtweiß, C. Dietrich, W. Zhang, J. O. Binder, P. Hartmann, W. G. Zeier, J. Janek, Chem. Mater. 2017, 29, 5574.

[46] H. Visbal, S. Fujiki, Y. Aihara, T. Watanabe, Y. Park, S. Doo, J. Power Sources 2014, 269, 396.

[47] H. Visbal, Y. Aihara, S. Ito, T. Watanabe, Y. Park, S. Doo, J. Power Sources 2016, 314, 85.

[48] Y. Seino, T. Ota, K. Takada, A. Hayashi, M. Tatsumisago, Energy Environ. Sci. 2014, 7, 627.

[49] X. Chen, W. He, L.-X. Ding, S. Wang, H. Wang, Energy Environ. Sci. 2019, 12, 938.

[50] Z. Zhang, Y. Zhao, S. Chen, D. Xie, X. Yao, P. Cui, X. Xu, J. Mater. Chem. A 2017, 5, 16984.

[51] N. C. Rosero-Navarro, A. Miura, K. Tadanaga, J. Power Sources 2018 396,33

[52] H. Kitaura, A. Hayashi, T. Ohtomo, S. Hama, M. Tatsumisago J. Mater. Chem. 2011, 21, 118.

[53] Y. Su, G. Chen, L. Chen, Y. Lu, Q. Zhang, Z. Lv, C. Li, L. Li, N. Liu, G. Tan, L. Bao, S. Chen, F. Wu, ACS Appl. Mater. Interfaces 2019, 11, 36697.

[54] P. Zou, Z. Lin, M. Fan, F. Wang, Y. Liu, X. Xiong, Appl. Surf. Sci. 2020, 504, 144506

[55] Y.-Y. Wang, Y.-Y. Sun, S. Liu, G.-R. Li, X.-P. Gao, ACS Appl. Energy Mater. 2018, 1, 3881.

[56] D. Ren, Y. Yang, L. Shen, R. Zeng, H. D. Abruña, J. Power Sources 2020, 447, 227344.

[57] D. Becker, M. Börner, R. Nölle, M. Diehl, S. Klein, U. Rodehorst, R. Schmuch, M. Winter, T. Placke, ACS Appl. Mater. Interfaces 2019, 11,18404

[58] S.-J. Sim, S.-H. Lee, B.-S. Jin, H.-S. Kim, Sci. Rep. 2019, 9, 8952.

[59] S. Choi, I. Yoon, W. T. Nichols, D. Shin, Ceram. Int. 2018, 44, 7450.

[60] Y. Ito, M. Otoyama, A. Hayashi, T. Ohtomo, M. Tatsumisago, J. Power Sources 2017, 360, 328.

[61] J. H. Woo, J. E. Trevey, A. S. Cavanagh, Y. S. Choi, S. C. Kim, S. M. George, K. H. Oh, S.-H. Lee, J. Electrochem. Soc. 2012, 159, A1120.

[62] Y. Seino, T. Ota, K. Takada, J. Power Sources 2011, 196, 6488.

[63] N. Ohta, K. Takada, I. Sakaguchi, L. Zhang, R. Ma, K. Fukuda, M. Osada, T. Sasaki, Electrochem. Commun. 2007, 9, 1486.

[64] X. Hao, Q. Zhao, S. Su, S. Zhang, J. Ma, L. Shen, Q. Yu, L. Zhao, Y. Liu, F. Kang, Y.-B. He, Adv. Energy Mater. 2019, 9, 1901604.

[65] J. Janek, W. G. Zeier, Nat. Energy 2016, 1, 16141.

[66] Y. Xiao, L. J. Miara, Y. Wang, G. Ceder, Joule 2019, 3, 1252.

[67] K. Yoon, J. J. Kim, W. M. Seong, M. H. Lee, K. Kang, Sci. Rep. 2018, 8.

[68] H. Nakamura, T. Kawaguchi, T. Masuyama, A. Sakuda, T. Saito, K. Kuratani, S. Ohsaki, S. Watano, J. Power Sources 2020, 448, 227579.

[69] D. H. Kim, D. Y. Oh, K. H. Park, Y. E. Choi, Y. J. Nam, H. A. Lee, S.-M. Lee, Y. S. Jung, Nano Lett. 2017, 17, 3013. 